\title{
STRING TENSION AND THE GENERATION OF THE CONFORMAL ANOMALY
}

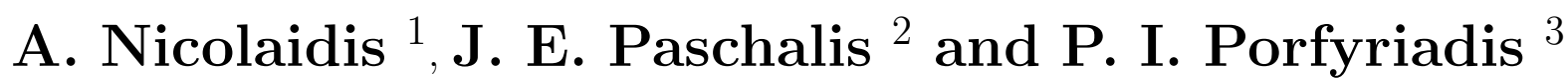 \\ Department of Theoretical Physics, University of Thessaloniki, Thessaloniki 54006, \\ Greece
}

\begin{abstract}
The origin of the string conformal anomaly is studied in detail. We use a reformulated string Lagrangian which allows to consider the string tension $T_{0}$ as a small perturbation. The expansion parameter is the worldsheet speed of light $\mathrm{c}$, which is proportional to $T_{0}$. We examine carefully the interplay between a null (tensionless) string and a tensionful string which includes orders $c^{2}$ and higher. The conformal algebra generated by the constraints is considered. At the quantum level the normal ordering provides a central charge proportional to $c^{2}$. Thus it is clear that quantum null strings respect conformal invariance and it is the string tension which generates the conformal anomaly.
\end{abstract}

\footnotetext{
${ }^{1}$ nicolaid@ccf.auth.gr

2 paschali@ccf.auth.gr

${ }^{3}$ porfyriadis@physics.auth.gr
} 
The most striking feature of quantum string models is their prediction of a critical spacetime dimension outside of which the quantum theory is problematical. Clearly it is quite important to understand how conformal invariance is broken by quantum effects. The whole issue becomes even more interesting by noticing the similarities between the canonical formulations of the string model and of general relativity. In both cases the Hamiltonian is weakly vanishing and the existing constraints reflect the underlying reparametrization invariance ( in two or four dimensions ) [1]. It appears that we should address the problem within a framework which allows us to consider the string tension as a small perturbation. Such a formalism, the $c$ expansion, has been proposed by De Vega and Nicolaidis in ref.[2]. The limit $T_{0} \rightarrow 0$ corresponds to the high energy limit of the string theory and it is also the appropriate limit for a string in strong gravitational field. The zeroth term in the $c$ expansion provides the null string. The null string is a tensionless string where each point of the string moves along a null geodesic [3]. The quantization of a null string in de Sitter spacetime was studied in ref.[4]. No anomaly was found and it was conjectured that the dimensionful string tension lies at the origin of the conformal anomaly. We examine carefully here the quantum generation of the conformal anomaly and trace its explicit dependence upon the string tension. Aspects of this problem were addressed also in [5].

The starting point is the reformulated string Lagrangian [2]

$$
L=\frac{1}{4 \lambda}\left[\dot{X}^{\mu} \dot{X}^{\nu} G_{\mu \nu}(X)-c^{2} X^{\prime \mu} X^{\prime \nu} G_{\mu \nu}(X)\right]
$$

where $c=2 \lambda T_{0}$ is the world-sheet speed of light. The string equations of motion read

$$
\ddot{X}^{\mu}-c^{2} X^{\prime \prime \mu}+\Gamma_{\kappa \lambda}^{\mu}\left(\dot{X}^{\kappa} \dot{X}^{\lambda}-c^{2} X^{\prime \kappa} X^{\prime \lambda}\right)=0
$$

supplemented by the constraints

$$
\begin{aligned}
& \Phi=\frac{1}{2}\left(\dot{X}^{\mu} \dot{X}^{\nu} G_{\mu \nu}+c^{2} X^{\prime \mu} X^{\prime \nu} G_{\mu \nu}\right)=0 \\
& \Psi=c \dot{X}^{\mu} X^{\prime \nu} G_{\mu \nu}=0
\end{aligned}
$$

Now we proceed to a series expansion in powers of $c$ [2]. Writing

$$
X^{\mu}(\sigma, \tau)=A^{\mu}(\sigma, \tau)+c^{2} B^{\mu}(\sigma, \tau)+\cdots
$$

we obtain the equations of motion for the zeroth order term

$$
\ddot{A}^{\mu}+\Gamma_{\kappa \nu}^{\mu} \dot{A}^{\kappa} \dot{A}^{\nu}=0
$$

and the constraints

$$
\begin{aligned}
\phi & =\frac{1}{2} \dot{A}^{\mu} \dot{A}^{\nu} G_{\mu \nu}=0 \\
\psi & =c \dot{A}^{\mu} A^{\prime \nu} G_{\mu \nu}=0
\end{aligned}
$$


$A^{\mu}(\sigma, \tau)$ represents a tensionless string, a collection of massless particles moving independently along null geodesics. For a string in Minkowski spacetime the null string solution is 3

$$
A^{\mu}(\sigma, \tau)=q^{\mu}(\sigma)+p^{\mu}(\sigma) \tau
$$

and it is obtained from the full string solution by keeping terms constant and linear in $c \tau$. String tension corrections appear when terms proportional to $(c \tau)^{2},(c \tau)^{3} \ldots$ are included.Thus $c$ expansion amounts to a short time expansion for the internal motion, while the overall string motion in the external geometry is treated exactly. In this sense $c$ expansion is an improvement over short time expansions proposed earlier [6, 7].

Under the Poisson bracket

$$
\left\{P^{\mu}(\sigma), X^{\nu}\left(\sigma^{\prime}\right)\right\}=\delta\left(\sigma-\sigma^{\prime}\right) G^{\mu \nu}
$$

the constraints generate the conformal algebra ( we set $2 \lambda=1$ )

$$
\begin{aligned}
& \left\{\Phi(\sigma), \Phi\left(\sigma^{\prime}\right)\right\}=c\left[\Psi(\sigma)+\Psi\left(\sigma^{\prime}\right)\right] \delta^{\prime}\left(\sigma-\sigma^{\prime}\right) \\
& \left\{\Phi(\sigma), \Psi\left(\sigma^{\prime}\right)\right\}=c\left[\Phi(\sigma)+\Phi\left(\sigma^{\prime}\right)\right] \delta^{\prime}\left(\sigma-\sigma^{\prime}\right) \\
& \left\{\Psi(\sigma), \Psi\left(\sigma^{\prime}\right)\right\}=c\left[\Psi(\sigma)+\Psi\left(\sigma^{\prime}\right)\right] \delta^{\prime}\left(\sigma-\sigma^{\prime}\right)
\end{aligned}
$$

Notice that the algebra, eqs.(11), is valid for any spacetime metric $G_{\mu \nu}$. The constraints $\Psi$ and $\Phi$ generate two-dimensional reparametrizations of the string history $X^{\mu}(\sigma, \tau)$. Indeed the generator

$$
F=-\int\left(\xi_{0} \Phi+\xi_{1} \Psi\right) d \sigma
$$

changes $X^{\mu}(\sigma, \tau)$ by

$$
\delta X^{\mu}(\sigma, \tau)=\left\{X^{\mu}(\sigma, \tau), F\right\}=\xi_{0} \dot{X}^{\mu}(\sigma, \tau)+\xi_{1} X^{\prime \mu}(\sigma, \tau)
$$

The above transformation is identical to the one induced through infinitesimal changes of the world-sheet coordinates $\tau, \sigma$ by $\xi_{0}, \xi_{1}$ respectively. Following the rule that null string results are obtained by dropping terms proportional to $c^{2}, c^{3} \ldots$, we obtain from eqs.(11), the algebra for a null string

$$
\begin{aligned}
\left\{\phi(\sigma), \phi\left(\sigma^{\prime}\right)\right\} & =0 \\
\left\{\phi(\sigma), \psi\left(\sigma^{\prime}\right)\right\} & =c\left[\phi(\sigma)+\phi\left(\sigma^{\prime}\right)\right] \delta^{\prime}\left(\sigma-\sigma^{\prime}\right) \\
\left\{\psi(\sigma), \psi\left(\sigma^{\prime}\right)\right\} & =c\left[\psi(\sigma)+\psi\left(\sigma^{\prime}\right)\right] \delta^{\prime}\left(\sigma-\sigma^{\prime}\right)
\end{aligned}
$$

For open string, the Fourier components of the constraints

$$
\begin{aligned}
& \Phi_{n}=\frac{2}{\pi} \int_{0}^{\pi} \Phi(\sigma) \cos n \sigma d \sigma \\
& \Psi_{n}=\frac{2}{\pi} \int_{0}^{\pi} \Psi(\sigma) \sin n \sigma d \sigma
\end{aligned}
$$


satisfy the algebra

$$
\begin{aligned}
& \left\{\Phi_{n}, \Phi_{m}\right\}=-\frac{c}{\pi}(m-n) \Psi_{n+m} \\
& \left\{\Phi_{n}, \Psi_{m}\right\}=\frac{c}{\pi}(m-n) \Phi_{n+m} \\
& \left\{\Psi_{n}, \Psi_{m}\right\}=\frac{c}{\pi}(m-n) \Psi_{n+m}
\end{aligned}
$$

and similarly for the null string case, from eqs.(14), we obtain for the corresponding Fourier components

$$
\begin{aligned}
\left\{\phi_{n}, \phi_{m}\right\} & =0 \\
\left\{\phi_{n}, \psi_{m}\right\} & =\frac{c}{\pi}(m-n) \phi_{n+m} \\
\left\{\psi_{n}, \psi_{m}\right\} & =\frac{c}{\pi}(m-n) \psi_{n+m}
\end{aligned}
$$

Moving from the classical to the quantum domain we replace the Poisson bracket by the commutator

$$
\left[P^{\mu}(\sigma), X^{\nu}\left(\sigma^{\prime}\right)\right]=i \delta\left(\sigma-\sigma^{\prime}\right) G^{\mu \nu}
$$

The ordering of the operators offers the possibility for the emergence of central charges in the algebra. The appropriate ordering may be found by studying the quantum ground state. A string in Minkowski spacetime can be viewed as an ensemble of harmonic oscillators and normal ordering (annihilation operators to the right of creation operators) is the appropriate one. Writing for an open string in Minkowski spacetime

$$
X^{\mu}(\sigma, \tau)=q_{0}^{\mu}+\frac{1}{\pi} \Pi^{\mu} \tau+\sum_{n=1}^{\infty} X_{n}^{\mu}(\tau) \cos n \sigma
$$

we define the oscillator variables by

$$
\begin{aligned}
\alpha_{n}^{\mu} & =\frac{1}{2}\left(\dot{X}_{n}^{\mu}-i n c X_{n}^{\mu}\right) \\
\alpha_{n}^{\mu *} & =\frac{1}{2}\left(\dot{X}_{n}^{\mu}+i n c X_{n}^{\mu}\right)
\end{aligned}
$$

In terms of the new variables $\left(q_{0}^{\mu}, \Pi^{\mu}, \alpha_{n}^{\mu}, \alpha_{n}^{\mu *}\right)$ the Fourier coefficients of the constraints take the form

$$
\begin{aligned}
& \Phi_{n}=\frac{1}{\pi} \Pi\left(\alpha_{n}+\alpha_{n}^{*}\right)+\frac{1}{2} \sum_{m=1}^{n-1}\left(\alpha_{m} \alpha_{n-m}+\alpha_{m}^{*} \alpha_{n-m}^{*}\right)+\sum_{m=1}^{\infty}\left(\alpha_{m}^{*} \alpha_{n+m}+\alpha_{n+m}^{*} \alpha_{m}\right) \\
& \Psi_{n}=-\frac{i}{\pi} \Pi\left(\alpha_{n}-\alpha_{n}^{*}\right)-\frac{i}{2} \sum_{m=1}^{n-1}\left(\alpha_{m} \alpha_{n-m}-\alpha_{m}^{*} \alpha_{n-m}^{*}\right)-i \sum_{m=1}^{\infty}\left(\alpha_{m}^{*} \alpha_{n+m}-\alpha_{n+m}^{*} \alpha_{m}\right)
\end{aligned}
$$


Eq.(18) provides the following commutators

$$
\begin{aligned}
{\left[\Pi^{\mu}, q_{0}^{\nu}\right] } & =i G^{\mu \nu} \\
{\left[\alpha_{n}^{\mu}, \alpha_{m}^{\nu *}\right] } & =-c \frac{n}{\pi} \delta_{n m} G^{\mu \nu}
\end{aligned}
$$

The quantum algebra generated by the constraints is evaluated using the normal ordering. After some lengthy calculations we obtain

$$
\begin{aligned}
& {\left[\Phi_{n}, \Phi_{m}\right]=\frac{i c}{\pi}(n-m) \Psi_{n+m}} \\
& {\left[\Phi_{n}, \Psi_{m}^{*}\right]=\frac{i c}{\pi}(n+m) \Phi_{n-m}+\frac{i d}{6 \pi^{2}} c^{2}\left(m^{3}-m\right) \delta_{n m}} \\
& {\left[\Psi_{n}, \Phi_{m}^{*}\right]=-\frac{i c}{\pi}(n+m) \Phi_{n-m}-\frac{i d}{6 \pi^{2}} c^{2}\left(m^{3}-m\right) \delta_{n m}} \\
& {\left[\Psi_{n}, \Psi_{m}\right]=-\frac{i c}{\pi}(n-m) \Psi_{n+m}}
\end{aligned}
$$

The above quantum algebra is the main result of our work. The anomaly which appears in (25) and (26), is of order $c^{2}$. The anomaly originates from the reordering of the operators, each reordering providing a factor $c$ (eq.(23)). The actual calculation indicates that the anomaly stems from the commutator $\left[\alpha_{k} \alpha_{n-k}, \alpha_{l}^{*} \alpha_{n-l}^{*}\right]$ (and its hermitean congugate), where eq.(23) is applied twice. Imagine a string with a vanishing small tension $T_{0}$. Our calculation shows that the anomaly vanishes also as $T_{0}^{2}$. The fact that the string tension generates the conformal anomaly has been indicated also earlier [8].

The transition from a quantum tensionful string to a quantum tensionless string is not a continuous process. In the case $T_{0}=0, \alpha_{n}^{\mu}$ and $\alpha_{n}^{\mu *}$ become identical and they commute. The appropriate ordering then is to place the momentum operators $\left(P_{n}=\frac{1}{2 \lambda} \dot{X}_{n}\right)$ to the right of the position operators $\left(X_{n}\right)$. The following quantum algebra ( without any central charge) is obtained for the null string

$$
\begin{aligned}
{\left[\phi_{n}, \phi_{m}\right] } & =0 \\
{\left[\phi_{n}, \psi_{m}^{*}\right] } & =\frac{i c}{\pi}(n+m) \phi_{n-m} \\
{\left[\psi_{n}, \phi_{m}^{*}\right] } & =-\frac{i c}{\pi}(n+m) \phi_{n-m} \\
{\left[\psi_{n}, \psi_{m}\right] } & =-\frac{i c}{\pi}(n-m) \psi_{n+m}
\end{aligned}
$$

It is important to emphasize that the same algebra is obtained from the full quantum algebra, eqs. (24)-(27), following the rule we proposed, i.e. dropping terms $c^{2}$ and higher. f

Summarizing, we studied the emergence of the string conformal anomaly. We introduced a reformulated Lagrangian which allows to consider the limit $T_{0} \rightarrow 0$. We have

\footnotetext{
${ }^{4}$ Regarding the quantization of a null string, an ordering prescription not implied by the physical problem itself, may lead to the emergence of an apparent quantum anomaly [9]
} 
shown that the conformal anomaly is proportional to $T_{0}^{2}$ and vanishes for a tensionless string. Throughout and at any stage, we are able to move from the full theory to the tensionless case (null string), via the correspondence rule we have established. Our explicit calculation demonstrates also the importance of the correct ordering of the quantum operators. We are familiar with the breaking of gauge invariance by a mass term and how this is cured through the Higgs mechanism. We may contemplate for a mechanism generating string tension and at the same time respecting the conformal symmetry.

The authors wish to acknowledge many useful discussions with Hector de Vega. This work was supported in part by the General Secretariat of Research and Technology (Greece) and the EU program "Human Capital and Mobility".

\section{References}

[1] M. Henneaux, in Principles of String Theory, Plenum Press, NY(1988).

[2] H. J. de Vega and A. Nicolaidis, Phys. Lett. 259B, 214 (1992)

H. J. de Vega and A. Nicolaidis, c expansion for strings, to appear in the Proceedings of the Network Meeting "String Gravity", Paris, June 1996.

[3] A. Schild, Phys.Rev. D16, 1722 (1977)

A. Karlhede and U. Lindstrom, Class.Quantum Grav. 3 , L73 (1986).

[4] H. J. de Vega, I. Giannakis and A. Nicolaidis, Mod. Phys. Lett. A10, 2479 (1995).

[5] F. Lizzi, Mod. Phys. Lett. A9, 1495 (1994).

[6] N. Sanchez and G. Veneziano, Nucl. Phys. B333, 253 (1990).

[7] M. Gasperini, N. Sanchez and G. Veneziano, Int. J. Mod. Phys. A16, 3853 (1991) and Nucl. Phys. B364, 365 (1991).

[8] L. Brink and H. Nielsen, Phys. Lett. B45 , 332 (1973).

[9] F. Lizzi et al., Phys. Lett. B182 , 326 (1986)

J. Gamboa, C. Ramirez and M. Ruiz-Altaba, Nucl. Phys. B338 , 143 (1990)

J. Isberg et al., Nucl. Phys. B411 , 122 (1994)

H. Gustafsson et al., Nucl. Phys. B440 , 495 (1995)

I. Bandos, A. Zheltukhin, Sov. J. Nucl. Phys. 50, 556 (1989). 